\title{
ON THE SUBRING GENERATED BY THE SYMMETRIC ELEMENTS
}

\author{
W. E. BAXTER ${ }^{1}$
}

J. M. Osborn [3] has characterized those rings with involution $J$, with 1 , and such that every symmetric element has an inverse under the additional hypothesis that $S^{-}$, the subring generated by $S$ (the set of symmetric elements), is $A$, the ring itself. In this paper we raise the question as to when $S^{-}=A$ under a weaker hypothesis and prove the following theorem. ( $A$ henceforth is such that $2 A=A$ and $2 x=\theta$ implies $x=\theta$ for any $x \in A$.)

TheOREM 1. Let $A$ be a ring with 1 and suppose that $S$ is a simple Jordan ring under the Jordan multiplication, $s \circ t=s t+t s$ for all $s$ and $t$ in $S$. Then either $S^{-}=A$ or $S \subset Z$, the center of $A$, or $K$, the set of skew elements, is an ideal of $A$ with $K^{2} \subset Z, K^{3}=(\theta)$, and $S$ is an associative ring under Jordan multiplication.

We note that Osborn proves that under his hypothesis, either $A$ is a division ring; the direct sum of two division rings which are antiisomorphic, and $J$ interchanges the summands; or $S$ is a field under Jordan multiplication, $K$ is an ideal of $A$ where $K^{2}=(\theta)$. A corollary to our result is

Corollary 1. Let $A$ be as in Theorem 1. Suppose further that $A$ contains no nilpotent ideals. Then either $A$ is simple or $A$ is a direct sum of two simple rings which are anti-isomorphic and such that $J$ interchanges the direct summands.

In order to prove these results we note quickly

Lemma 1. If $U$ is a proper ideal of $A$ then $U \cap S=(\theta)$.

This follows since $S \cap U$ is a Jordan ideal of $S$ and hence if not zero is $S$ itself. But $1 \in S$ implies that $U=A$.

We are ready to prove Theorem 1 . Herstein [1] has shown that $S^{-}$ is a Lie ideal of $A$ and Zuev [4] has shown that either $S^{-}$is commutative (in particular $[S, S]=(\theta)$ ) or $I=\left\{u \mid u \in S^{-}, u a \in S^{-}\right.$for all $\left.a \in A\right\}$ is a nonzero two-sided ideal of $S^{-}$. We next note that $[S, S] \subseteq I$. To

Received by the editors March 30, 1967.

1 The author wishes to express his gratitude to the Department of Mathematics, University of Oregon for the many kindnesses shown him during the year when this manuscript was prepared. 
observe this fact, let $s, t, u \in S, k \in K$; then most certainly $(s t-t s) u$ $\in S^{-}$, while $[s, t] k=s[t, k]+[s, k] t+\{k s t-t s k\}$. As the last quantity is in $S$ we have our desired conclusion. Moreover, $[S, S] \subseteq V=I \cap I^{J}$. Thus, by Lemma 1 we have either $V=A$ and hence $A=I \subset S^{-}$(part of our desired conclusion) or $V \cap S=(\theta)$. So far we have shown that either $S^{-}=A,[S, S]=(\theta)$, or $[S, S] \subseteq V$, where $V$ is an ideal such that $V \cap S=(\theta)$.

We will have almost proved Theorem 1 if we can show that the latter situation $\left([S, S] \subseteq V, V\right.$ an ideal with $V=V^{J}$ and $V \cap S=(\theta)$ ) implies either $[S, S]=(\theta)$ or the latter possibility in the consequence of the theorem. We now restrict ourselves to this case.

If $u \in V$ then there exists $v \in V$ so that $u=v^{J}$. Thus, as a consequence of $V \cap S=(\theta)$ we have $u+v=u+u^{J}=\theta$, or $V \subseteq K$. $V$ is an ideal and so for all $a \in A, u \in V, u a-a^{J} u=\theta$ (in particular, $[S, S] \circ K$ $=(\theta))$. This guarantees that $[S, K]$ is a Jordan ideal of $S$ since $[S, K]$ $\circ S \subseteq K \circ[S, S]+[S, K]$. Thus, the simplicity of $S$ implies that either $[S, K]=S$ or $[S, K]=(\theta)$.

We now see that the subcase $[S, K]=S$ leads to $[S, S]=(\theta)$. To this end, consider $a, b \in A, v \in V$. Then,

$$
\theta=v a b-(a b)^{J} v=\left(v a-a^{J} v\right) b+a^{J}\left(v b-b^{J} v\right)+\left(a^{J} b^{J}-b^{J} a^{J}\right) v
$$

or

$$
[A, A] V=(\theta) \text {. }
$$

In particular, $[S, K][S, S]=(\theta)$ and so $[S, K]=S$ and $1 \in S$ yields $[S, S]=(\theta)$.

Thus, the subcase $[S, K]=(\theta)$ remains. Here we show that either $S \subset Z$ or the latter alternative of the theorem holds. In this case we have $\left[k^{2}, s+l\right]=k \circ[k, s]+[k, k \circ l]=\theta$ for all $k, l \in K, s \in S$. Thus, $k^{2} \in Z$ for all $k \in K$. Therefore, $k l+l k=(k+l)^{2}-k^{2}-l^{2} \in Z$ for all $k, l \in K$ or $K \circ K \subseteq Z$. Now, $[K, S]+K \circ K$ is a Jordan ideal of $S$. However, this is just $K \circ K$ under our hypothesis. Thus, either $K \circ K=S \subseteq Z$ (a desired conclusion) or $K \circ K=(\theta)$. The latter situation now concerns us. Now for all $a \in A, k \in K, k a-a^{J} k \in[S, K]$ $+K \circ K=(\theta)$. Thus, $k, l \in K, a \in A$ implies $[k l, a]=k\left(l a-a^{J} l\right)$ $+\left(k a^{J}-a k\right) l=\theta$, or $K^{2} \subset Z$. Under our assumptions we also note that $K A \subseteq K \circ S+[K, K] \subseteq K$; that is, $K$ is an ideal of $A . K \circ K=(\theta)$ implies that $k^{2}=\theta$ and $k \circ l=\theta$ for all $k, l \in K$. Thus, $k l k=\theta$ and replacing $k$ by $k+m, m$ also in $K$, we have $k l m+m l k=\theta$. But $K$ is an ideal, so $(m l) k+k(m l)=m l k-k l m=\theta$. Hence, $K^{3}=(\theta)$. Now for all $s, t, u \in S,(s \circ t) \circ u=s \circ(t \circ u)+[t,[s, u]]$. The latter term being 
zero implies that $S$ is an associative ring under the Jordan multiplication.

Therefore, we have shown that either $S^{-}=A,[S, S]=(\theta)$, or the third consequence in the statement of the theorem holds. What remains is to show that $[S, S]=(\theta)$ implies $S \subseteq Z$. Fix $s \in S$ and consider $[s, K]$. For all $k \in K, t \in S$

$$
[s, k] \circ t=[s, k \circ t]-k \circ[s, t] \text {. }
$$

By hypothesis the latter term is zero and so $[s, K]$ is a Jordan ideal of $S$. Now, if $[s, K]=(\theta)$ for each $s \in S$ then $S \subseteq Z$ (as $[S, S]=(\theta)$ ). We show that the other alternative, $[u, K]=S$ for some $u \in S$ leads to a contradiction. As $[S, S]=(\theta)$ we conclude that $[u,[u, a]]=\theta$ for all $a \in A$. Therefore, replacing $a$ by $a b$ and expanding out (using the fact that $2 x=\theta$ implies $x=\theta$ ) we obtain for all $a, b \in A$

$$
(u a-a u)(u b-b u)=\theta .
$$

Since $1 \in S$ and $[S, S]=(\theta), S^{2}=S$ and so $[u, K]^{2}=S^{2}=S=(\theta)$, a contradiction. Therefore, $S \subseteq Z$ as desired.

The proof of Corollary 1 follows. We first note that the additional hypothesis on $A$ implies that either $S^{-}=A$ or $S \subseteq Z$. Now, if $A$ is not simple then we wish to show that the alternative of the conclusion holds. Let $U$ be a proper nonzero ideal of $A$. It readily follows that $T=\left\{u+u^{J} \mid u \in U\right\}$ is a Jordan ideal of $S$. Now, if $T \neq(\theta)$ then $S=T$ or $A=U+U^{J}$. We show that indeed the summation is direct and $U$ is a simple ring. By Lemma $1, U \cap S=(\theta)$. Hence, $V=U \cap U^{J}$ is an ideal with the property $V \cap S=(\theta)$ as well. As before, we conclude that $V \subseteq K$, and $V^{3}=(\theta)$. Hence, $V=(\theta)$ or the summation is direct. Now, let $W \neq(\theta)$ be an ideal of $U$. Then, $R=U W U$ is an ideal of $A$, contained in $U$, and nonzero. Else, $(A R)^{3} \subseteq U W U=(\theta)$, and this is a contradiction to no nilpotent ideals by Herstein [1]. As before, $A=R+R^{J}$. Therefore, $U \subseteq R \subseteq W$ as $W^{J} \subseteq U^{J}$. Thus, $U$ is simple as desired. Hence, if we show $T=(\theta)$ is impossible we are done. Now, the latter implies that $U \subseteq K$, and analogous to a previous argument $U^{3}=(\theta)$. But the additional hypothesis forcing $U=(\theta)$ makes this impossible.

Corollary 2. Let $A$ be without nilpotent ideals. Then either $S^{-}=A$ or $A$ is simple and $[A: Z] \leqq 4$ or $A=U+U^{J}$, where $U$ is simple and $[U: Z] \leqq 4$.

We have shown in Theorem 1 that either $S^{-}=A$ or $S \subseteq Z$. Now suppose the latter. Then given any $r \in A$ there exists $s \in S, k \in K$ so 
that $r=s+k$. Thus $r^{2}-2 s r=k^{2}-s^{2} \in Z$, or each element in $A$, satisfies a quadratic equation over $Z$. The latter, together with Corollary 1 , by the work of Kaplansky [2], yields the desired conclusion.

In addition if $A$ is prime then $A$ cannot be written as $U+U^{J}$ and so $A$ is simple.

We assume $1 \notin A$. Then certain of the results carry over and others do not. The absence of 1 does not allow us to conclude that if $U$, an ideal, is such that $U \supseteq S$ then $U=A$. However, we do have in this situation that both $a+a^{J}$ and $a a^{J}$ are in $U$ for all $u \in A$. Thus $a^{2} \in U$ for all $a \in A$, or $A^{3} \subseteq U$ (for example, $A^{2}=A$ implies that $A=U$ ). On the other hand, if $U \cap S=(\theta)$, then $V=U \cap U^{J} \subseteq K$ and $V^{3}=(\theta)$. $T=\left\{u+u^{J} \mid u \in U\right\}$ is either $(\theta)$ or $S$. If $T=(\theta)$ then $U \subseteq K$ and $U^{3}=(\theta)$; while $T=S$ implies that $A^{3} \subseteq U+U^{J}$. Now, if we restrict our attention to the ideal $I$ as defined previously in relation to $T$ we have either $S \subseteq I+I^{J} \subseteq S^{-}$, which says that $S^{-}$is an ideal or $T=(\theta)$; that is, $I \subseteq K, I \cap S=(\theta)$, and $I^{3}=(\theta)$. As in the proof of Theorem 1 this implies that either $[S, K]=S$ or $[S, K]=(\theta)$. As before, $[S, K]$ $=S$ yields $[S, S] S=S[S, S]=(\theta)$ and $[S, S] K \subseteq[S, S] \circ K$ $+[[S, S], K] \subseteq[S, S]($ as $[S, S] \circ K=(\theta))$. Thus, in this case, $[S, S]$ is an ideal with $[S, S]^{3}=(\theta)$. On the other hand, $[S, K]=(\theta)$ yields the same argument as before. We summarize these remarks as

THEOREM 2. Let $A$ be a ring with involution and suppose that $S$ is simple Jordan. Then either $S^{-}$is an ideal (of $A$ ) containing $A^{3}$, or $[S, S]$ is an ideal with $[S, S]^{3}=(\theta)$, or $K$ is an ideal, $K^{2} \subseteq Z, K^{3}=(\theta)$ and $S$ is an associative ring under Jordan multiplication.

Now assume that there are no nilpotent ideals in $A$; then we have either $S^{-}$is an ideal or $[S, S]=(\theta)$. The latter yields, as in a previous argument, that for all $a, b \in A, u \in S$,

$$
(u a-a u)(u b-b u)=(\theta),
$$

and replacing $b$ by $b a$ and expanding we have $\{(u a-a u) A\}^{2}=(\theta)$. But by the hypothesis and Herstein [1] we conclude that $S \subseteq Z$. Thus, either $S^{-}$is an ideal or $S \subseteq Z$ and every $a \in A$ satisfies a quadratic equation over $Z$. Now if $U$ is any proper nonzero ideal, then $S$ $=\left\{u+u^{J} \mid u \in U\right\}$ and so $a+a^{J}=u+u^{J}$ for each $a \in A$ and suitable $u \in U$. Therefore $a-u \in K$ and so $A=U+K$. If $U \cap S=(\theta)$ then, under these hypotheses, $U \cap K=(\theta)$ and so the group sum is direct.

Finally, if $A$ is prime and $U$ is a nonzero ideal then $U$ contains $S$ (and hence the ideal $S^{-}$, unless $[S, S]=(\theta)$ ), as the other alternative $U \cap S=(\theta)$ implies $U \cap U^{J}=(\theta)$ or $U U^{J}=(\theta)$ which, by the hypothesis, is impossible. 


\section{REFERENCES}

1. I. N. Herstein, Topics in ring theory, Univ. of Chicago Math. Lecture Notes, 1965.

2. I. Kaplansky, Rings with a polynomial identity, Bull. Amer. Math. Soc. 54 (1948), 809-826.

3. J. M. Osborn, Jordan algebras of capacity two, Proc. Nat. Acad. Sci. U.S.A. 57 (1967), 582-588.

4. I. I. Zuev, Lie ideals of associative rings, Uspehi Mat. Nauk 18 (1963), no. 1 (109), 155-158.

University of Delaware 